DOI: https://doi.org/10.11144/Javeriana.upsy19.dccp

\title{
Diseño de un cuestionario de caracterización psicosocial y de motivos de consulta en Centros de Atención Psicológica Universitarios *
}

\section{Design of a Questionnaire for Psychosocial and Complaint Characterisation in University Psychological Assistance Centres}

\section{Tatiana Colón Llamas ${ }^{a}$}

Pontificia Universidad Javeriana, Colombia ORCID: https://orcid.org/0000-0002-9143-6007

Andrea Escobar Altare

Pontificia Universidad Javeriana, Colombia ORCID: https://orcid.org/0000-0002-7373-297X

Alejandro Granados-García

Pontificia Universidad Javeriana, Colombia ORCID: https://orcid.org/0000-0001-7201-4872

María Fernanda Parra-Murillo

Pontificia Universidad Javeriana, Colombia ORCID: https://orcid.org/0000-0002-9453-653X

Andrés Santacoloma Giraldo

Pontificia Universidad Javeriana, Colombia ORCID: https://orcid.org/0000-0003-4607-8025

SOCORRO MOREnO LUnA

Pontificia Universidad Javeriana, Colombia ORCID: https://orcid.org/0000-0002-4119-4409

Luis Manuel Silva Martin

Pontificia Universidad Javeriana, Colombia ORCID: https://orcid.org/0000-0001-7051-0186

a Autor de correspondencia. Correo electrónico: vcolon@javeriana.edu.co

Para citar este artículo: Colón-Llamas, T., EscobarAltare, A., Granados-García, A., Parra-Murillo, M. F., Santacoloma-Giraldo, A., Moreno-Luna, S., \& SilvaMartin, L. M. (2020). Diseño de un cuestionario de caracterización psicosocial y de motivos de consulta en Centros de Atención Psicológica Universitarios. Universitas Psychologica, 19, 1-12. https://doi.org/10.1 1144/Javeriana.upsy19.dccp

\section{RESUMEN}

Llevar a cabo la caracterización de los motivos de consulta en la atención psicológica suele ser difícil por su naturaleza variada y multidimensional y la consecuente falta de adecuados instrumentos para su sistematización. No obstante, hacerlo facilitaría la descripción de los principales motivos de consulta en psicología y la mejor orientación de los limitados recursos en la atención en salud mental. Este estudio descriptivo presenta el proceso de concepción, desarrollo y administración de un cuestionario de caracterización psicosocial y de motivos de consulta en una muestra de niños $\left(N=418, M_{\text {edad }}=8.4, D E_{\text {edad }}=2.17\right)$, adolescentes $(N=238$, $\left.M_{\text {edad }}=15.2, D E_{\text {edad }}=1.54\right)$ y adultos $\left(N=869, M_{\text {edad }}=30.8\right.$, $D E_{\text {edad }}=13.03$ ) de 28 Centros de Atención Psicológica Universitarios en Colombia y sus evidencias de validez, con el objetivo de brindar a la comunidad psicológica en Latinoamérica una guía para la construcción de instrumentos localizados. 
Palabras clave

motivos de consulta; atención psicológica; caracterización piscosocial; centro universitario de atención psicológica; cuestionarios.

\begin{abstract}
Characterizing chief psychological consulting complaints is often difficult due their varied and multidimensional nature, which is reflected by the lack of appropriate instruments to gather systematic information. However, such systematization would encourage better targeting of limited mental healthcare resources through a description and characterization of reasons for encounter. To add to these efforts, this descriptive study presents the process of conceptualizing, developing and applying a questionnaire to conduct a psychosocial and chief complaint characterization in a sample of children $(N=$ $\left.418, M_{\text {age }}=8.4, S D_{\text {age }}=2.17\right)$, teenagers $(N=238$, $\left.M_{\text {age }}=15.2, S D_{\text {age }}=1.54\right)$ and adults $(N=869$, Mage $\left.=30.8, \quad S D_{\text {age }}=13.03\right)$ who consulted at 28 University-based Psychological Assistance Centers across Colombia, along with validity evidences, with the goal of providing Latin American psychological communities with a guide to building localized instruments.

Keywords

reasons for consultation; psychological assistance; psychosocial characterization; university-based psychological assistance center; questionnaires.
\end{abstract}

La evaluación de la salud mental de la población en Colombia ha sido explorada con el fin de diseñar y promover políticas públicas (Ministerio de Salud y Protección Social \& Colciencias, 2015), identificar el panorama de la salud mental de los usuarios, establecer futuros tratamientos que, basados en la evidencia, se ajusten a las necesidades de la población y orientar la práctica clínica del psicólogo en formación (Narváez Marín \& Aguirre Loaiza, 2016).

No obstante, en muchos casos este intento se ha visto entorpecido por el acceso a los datos, pues, a pesar de existir sistemas de información provistos por el Ministerio de Salud como el Registro Individual de Prestación de Servicios de Salud (RIPS), gran parte de la información diligenciada no es confiable, debido a factores como la falta de precisión en la codificación por parte de los profesionales de la salud, la versatilidad y funcionalidad de las plataformas informáticas y la manipulación de los registros para su consolidación (Martínez y Pacheco (2013)). Como consecuencia, la información que daría cuenta del panorama de salud mental en el país es insuficiente para conocer las necesidades de la población y las dinámicas de la salud de forma integral, conduciendo a la dispersión de esfuerzos para la recolección de datos que puedan ser útiles para cualificar los servicios.

Dentro de estos esfuerzos se encuentran los desarrollados por De la Portilla Maya et al. (2016), Narváez Marín y Aguirre Loaiza (2016), Gómez Vargas y Pérez Jaramillo (2017), Obando-Posada et al. (2017), con el fin de describir las diferentes variables asociadas a las características sociodemográficas y a los motivos de consulta (MC) de sus centros de atención. En algunos países de Latinoamérica como Argentina, México y Costa Rica, existen investigaciones en las que se indaga y se relacionan factores sociodemográficos, psicosociales, criterios clínicos y una cierta sistematización de los MC (Espinosa et al., 2015; González-Villalobos \& Marrero, 2017; MarotoVargas et al., 2017; Quesada, 2004; Sosa et al., 2018).

La mayoría de estos estudios se han realizado en el marco de las Encuestas Nacionales de Salud Mental (ENSM) o en Instituciones Prestadoras de Salud (IPS) privadas. Los esfuerzos de los Centros de Atención Psicológica Universitarios, en lo relativo a la exploración de la población que consulta, son en general individuales (López Jaramillo et al., 2012; Narváez Marín \& Aguirre Loaiza, 2016; Obando-Posada et al., 2017), a pesar de que semestralmente alrededor de 10000 personas provenientes de diferentes regiones del país acuden a estos y requieren de los servicios que prestan. Según lo reportado por la Red de Instituciones de Servicios Universitarios de Atención Psicológica (ISUAP), en Colombia, los centros universitarios se presentan como una alternativa para la población, ante las limitaciones de atención en el Plan Obligatorio de Salud, debido a sus bajos costos, disponibilidad del servicio, calidad, duración, constancia y estabilidad en los tratamientos. Sin embargo, por esto mismo, sus agendas de atención se copan rápidamente y deben buscar ampliar su capacidad de cobertura y atención (Colón-Llamas et al., 2015). 
En consecuencia, y como antecedente para este trabajo, en 2007, la Red ISUAP propuso organizar los datos sociodemográficos, los MC y las expectativas de los consultantes que acuden a los Centros de Atención Psicológica Universitarios en una matriz que les permitiera sistematizar las problemáticas y dar cuenta de los motivos más frecuentes para solicitar ayuda, la cual fue utilizada por algunos centros para estudiar su población. (Moreno-Méndez et al., 2012)

Pese a los esfuerzos realizados, esta matriz dejaba un vacío en cuanto a los aspectos asociados al motivo de consulta, los cuales, en muchos casos, por ser de carácter psicosocial no eran contemplados como parte del mismo (Moreno-Méndez et al., 2012). Esto se convierte en un aspecto fundamental al tener en cuenta que Colombia ha tenido una prolongada historia de conflictos sociales y armados, cuyas distintas expresiones de violencia (social, política, económica y ambiental) han impactado directa e indirectamente la salud mental de la población, llevando a las personas a buscar atención y apoyo psicológico para aliviar su sufrimiento (Hurtado-Parrado et al., 2019; López López et al., 2018; Ministerio de Salud y Protección Social \& Colciencias, 2015; Pineda et al., 2003).

Por esta razón, entre 2017 y 2018, la Facultad de Psicología de la Pontificia Universidad Javeriana Sede Bogotá llevó a cabo una investigación en asocio con 27 centros de atención psicológica de las instituciones de la Red ISUAP, cuyo objetivo fue establecer una caracterización psicosocial y de los motivos de consulta de las personas que asisten por primera vez a estos centros universitarios en 16 ciudades del país. En el estudio, se indagó por diferentes factores psicosociales, debido a su importante incidencia en la salud mental del ser humano (Ministerio de Salud y Protección Social \& Colciencias, 2015).

Si bien la naturaleza de estos factores es heterogénea, varios autores han resaltado la importancia del grupo primario de apoyo, que en la mayoría de los casos es la familia (Ainsworth, 2000; Beckham \& Leber, 1997; Clark et al.,
1999). Otros factores de protección relevantes son los vínculos de calidad, las redes de apoyo, el nivel educativo y el uso adecuado del tiempo libre (Bezanilla et al., 2017).

Para la conducción de la investigación, se revisó extensamente la literatura en bases de datos internacionales (Web of Science y Scopus para artículos en inglés, Redalyc y SciELO para artículos en español y portugués), con el objetivo de buscar un instrumento que permitiera recoger la información de interés y, especialmente, la caracterización de los motivos de consulta de los consultantes. Los instrumentos encontrados se adherían a las nosologías tradicionales (DSM y CIE), y dejaban de lado la contextualización de los motivos de consulta. Por ejemplo, MiguelÁlvaro y Fernández-Arias (2019) examinaron la correspondencia entre la percepción del MC de pacientes y terapeutas, codificando motivos según DSM y experiencia clínica, pero se limitaron a los $\mathrm{MC}$ y no incluyeron aspectos psicosociales relacionados. Hosey et al. (2019) indagaron por interconsultas psicológicas en unidades de cuidado intensivo, pero nuevamente no incluyeron factores adicionales a los de MC, datos de hospitalización y tratamiento.

Por lo anterior, la primera fase de la investigación fue el diseño del instrumento de recolección. El propósito de este artículo es presentar el diseño y elaboración del protocolo e instrumento utilizado por los centros para caracterizar la población en términos psicosociales y sus motivos de consulta, con el fin de informar a la comunidad académica que empleó y seguirá empleando el instrumento y de servir de referencia para procesos similares en el futuro.

\section{Método}

\section{Diseño del estudio}

La construcción del instrumento hace parte de un estudio completo de diseño observacional, transversal, no experimental, cuyas principales conclusiones están descritas en el artículo "Caracterización psicosocial y de motivos de 
consulta de la población que asiste a 28 Centros de Atención Psicológica Universitarios en Colombia" (Colón-Llamas et al., 2019).

\section{Participantes}

La investigación contó con la participación voluntaria de 1525 personas, divididas en 418 niños (27.4 \%) entre 3 y 12 años; 238 adolescentes (15.6\%) entre 13 y 17 años y 869 adultos (56.9 \%) entre 18 y 80 años, de los cuales 811 (93 \%) consultaron de manera individual y 58 (7 \%) en pareja. Los participantes respondieron un cuestionario aplicado por profesionales y estudiantes de Psicología y Psiquiatría, de pregrado y posgrado, entre los meses de abril y agosto de 2018.

\section{Instrumento}

El instrumento cuenta con cuatro versiones: una para adultos (mayores de 18 años), otra para adolescentes (entre 13 y 17 años), otra para niños (entre 3 y 12 años, contestado por el acudiente) y una última para parejas adultas. A continuación, se presenta un recuento detallado de las fases de su construcción.

\section{Diseño del instrumento}

Inicialmente, se conformó un equipo de siete profesionales de una Facultad de Psicología, con experiencia en el campo clínico y psicosocial. Este equipo realizó una propuesta de cuatro ejes para indagación, con base en antecedentes del proyecto y la revisión bibliográfica pertinente de datos sociodemográficos, recursos psicosociales, riesgos psicosociales y motivos de consulta (Colón-Llamas et al., 2019).

Para validar la propuesta, se llevaron a cabo 10 grupos focales con los siete asesores con experiencia y uno con directores de Centros de las ciudades de Bogotá, Ibagué, Medellín, Barranquilla y Pereira. Los grupos focales se llevaron a cabo de manera presencial, en jornadas de trabajo de 2 a 4 horas, en las que se solicitó a los participantes que respondieran las siguientes preguntas: ¿Qué sugiere tener en cuenta al momento de realizar la caracterización psicosocial de la población consultante? y iqué sugiere tener en cuenta al momento de realizar la caracterización de los motivos de consulta de la población consultante? Como resultado de las jornadas de trabajo, se recogieron propuestas de preguntas y opciones de respuestas para las siguientes categorías: datos sociodemográficos, riesgo psicosocial, recursos psicosociales y motivos de consulta. Adicionalmente, se contó con la participación de asesores metodológicos de la Facultad de Psicología y del Departamento de Epidemiología Clínica de la Facultad de Medicina de la misma universidad. El objetivo de estos grupos fue diseñar las preguntas para la caracterización psicosocial de cada una de las poblaciones, dando como resultado un instrumento con cuatro versiones: niños, adolescentes, adultos y parejas, con preguntas de opción múltiple, una pregunta abierta (motivo de consulta) y campos de texto para profundizar la información.

Una vez finalizado el diseño, se envió a un grupo de cuatro expertos dentro de los que se contaba con profesionales en neuropsicología, psicología clínica, psicología social y medición y evaluación psicológica, para que hicieran observaciones narrativas sobre aspectos de pertinencia, relevancia, estructura gramatical y opciones de respuesta (EscobarPérez \& Cuervo-Martínez, 2008; Galicia Alarcón et al., 2017; Miller \& Lovler, 2020). De acuerdo con su retroalimentación, se modificó la redacción de algunos ítems para hacerlos más comprensibles o plantear algunas opciones de respuesta, específicamente, incluir la opción de "no responder" en varias de las preguntas. Cada cuestionario diligenciado contó con su correspondiente consentimiento informado, en el cual se explicaba el objetivo del proyecto, las medidas implementadas para la protección de sus derechos, riesgos y beneficios por su participación, y se aclaraban dudas o preguntas surgidas en el proceso. Lo anterior en cumplimiento con la Declaración de Helsinki en su versión más actualizada (World Medical 
Association, 2017) y la resolución 8430 de 1993 que clasifica la investigación en humanos en Colombia.

\section{Dimensiones}

A continuación, se describen cada una de las secciones para los cuatro ejes temáticos: datos sociodemográficos, recursos psicosociales, riesgos psicosociales y motivos de consulta e historia previa de atención, y los insumos utilizados en la recolección de información del cuestionario de Caracterización Psicosocial y de Motivos de Consulta.

\section{Datos sociodemográficos}

Incluye preguntas orientadas a conocer características propias de la persona y de su entorno, tales como: edad, género, orientación sexual, estado civil, etnia, creencias/actitudes religiosas, ocupación, nivel educativo, lugar de residencia, estrato socioeconómico, servicio de salud, régimen de salud, personas a cargo, personas con las que vive, condiciones económicas y características del entorno donde vive. Para esto, se utilizaron las escalas de datos sociodemográficos del Departamento Administrativo Nacional de Estadística (DANE) y de la ENSM de 2015.

Factores de protección psicosocial y redes de apoyo

Se entienden como aquellas condiciones, en las relaciones interpersonales o en el contexto del sujeto, que le previenen de sufrir excesivamente posibles desajustes sociales y emocionales (Colón-Llamas et al., 2019; Ministerio de Salud y Protección Social \& Colciencias, 2015).

Las preguntas buscaron conocer factores que pueden ser protectores para el consultante, tales como: redes de apoyo, vínculos y uso del tiempo libre. Para esto, se adaptaron y aplicaron los cinco ítems del APGAR sobre satisfacción familiar (Campo-Arias \& Caballero-Domínguez, en prensa; Smilkstein, 1978). Adicionalmente, se consideró importante, siguiendo a Bezanilla et al. (2017), incluir en el cuestionario para niños, preguntas sobre el tiempo libre y sus cuidadores principales, y en el instrumento de parejas, preguntas sobre qué valora de la pareja y las formas de resolución de conflictos entre ellos (Colón-Llamas et al., 2019).

\section{Factores de riesgo y estresores psicosociales}

Se entienden como aquellos elementos que aumentan la vulnerabilidad de una persona a padecer malestar emocional y desajustes psicosociales (Ministerio de Salud y Protección Social \& Colciencias, 2015). Se suelen asociar con precariedad en recursos económicos, sociales, emocionales, asistenciales y, en el caso particular del país, con las situaciones de violencia política, conflicto armado y crimen organizado presentes en numerosos sectores de la población (Corporación AVRE, 2002). Vale la pena diferenciarlos de los estresores psicosociales, que se refieren a situaciones demandantes y adversas propias de la interacción del sujeto con el medio (Londoño, Marín, Juárez, \& Palacio, 2010). Estos son, por ejemplo, problemas relativos al grupo primario o dificultades en el ambiente educativo, laboral, legal, etc. Los factores de riesgo en sí constituyen condiciones relativamente constantes en el medio en el que se desenvuelve el sujeto, como el maltrato físico, sexual o psicológico, el abandono real o emocional, la negligencia familiar o estatal y los patrones recurrentes en educación y crianza (Cáceres et al., 2006).

Para construir las preguntas de este eje, se convocó un panel de expertos que, con base en la bibliografía existente, diseñaron una serie de preguntas acerca de los riesgos a los que puede estar expuesto el consultante, y que son relativos a fenómenos tales como: situación familiar, violencia, conflicto armado, migración, pérdidas, enfermedad, discapacidad, consumo de sustancias, e ideación y conducta suicida. La estructura de estas preguntas fue verificada con posterioridad, tal como se describió anteriormente. 
Con el fin de identificar los factores de riesgo propios de cada etapa, se presentaron diferentes preguntas a cada grupo poblacional. En el caso del cuestionario de adultos y parejas, se incluyeron preguntas sobre si se presentaba maltrato físico, psicológico, sexual y/o patrimonial o económico; para parejas, se agregaron preguntas para conocer quién lo ejercía. En el cuestionario de niños se agregó un apartado relativo a dificultades o retrasos en el desarrollo y dificultades en diferentes áreas (alimentación, sueño, lenguaje, interacción con otros, juego, lectoescritura, rendimiento escolar y separación del cuidador). Para este cuestionario, también se creó una lista de chequeo de 28 síntomas, con el fin de identificar dificultades cognitivas, comportamentales y emocionales de los niños. Esta fue construida a partir de la escala de Pediatric Symptoms Checklist (Jellinek et al., 1988) y adaptada a las problemáticas evidenciadas en consulta en esta etapa del desarrollo, por los expertos. Algunos de los síntomas presentados fueron: dificultad para jugar, culpar a otros por sus problemas y dificultad para estar a solas.

En cuanto a los adolescentes, la lista de chequeo estuvo compuesta por 32 signos, donde se formulan preguntas relacionadas con problemáticas emocionales, de comportamiento, fenómenos propios de la adolescencia, autoagresión, problemas alimenticios, fenómenos psicóticos y otros relativos a la orientación y conducta sexual. Algunas de ellas fueron: manifiestas conductas de riesgo (consumo de alcohol y/o drogas, fugas, robos, accidentes repetidos), se siente aislado y se le dificulta comunicarse y ha experimentado cambios en el cuerpo que lo hacen sentirse extraño.

\section{Motivos de consulta}

Para obtener una imagen detallada del motivo de consulta del consultante, fue necesario indagar por la historia de su problemática, la duración e intensidad de la misma, las ideas que tiene sobre su problemática, el impacto que ha tenido en su vida, las expectativas asociadas, los intentos de solución y los tratamientos previos (Quesada, 2004).

El motivo de consulta fue definido como el malestar psicológico asociado a signos y síntomas y la dimensión afectiva que lleva a un sujeto a buscar alivio y una comprensión subjetiva de su sufrimiento; para la información específicamente relacionada con este, se dispuso de un campo donde se escribe el motivo de consulta, tal y como lo expresó el consultante o el acudiente. Sin embargo, para poder clasificar la información de los motivos de consulta fue necesario realizar dos procedimientos: una parte cuantitativa descriptiva de los subejes temáticos y una parte cualitativa basada en un análisis temático de los motivos de consulta registrados de manera literal.

Las categorías de análisis fueron producto de una triangulación entre expertos de la red ISUAP, los resultados de un análisis de frecuencia de palabras en el software Nvivo® en el cual fueron procesados 275 motivos de consulta de diferentes centros de atención psicológica del país de años anteriores y la revisión de literatura especializada. Esto derivó en la elaboración y definición de 27 categorías para el análisis de motivos de consulta.

Posteriormente, los profesores expertos realizaron una revisión por parejas para clasificar cada uno de los motivos de consulta recogidos en los 1525 cuestionarios. En esta revisión, emergieron cinco categorías de motivos asociados a: 1) dificultades en las relaciones paterno-filiales, 2) condiciones de discapacidad y situaciones de inclusión, 3) la exposición a situaciones traumáticas, 4) terceros y 5) motivos que no pueden ser categorizados por información insuficiente y otros, para un total de 31 categorías. 


\section{Tabla 1}

Categorización final de motivos de consulta

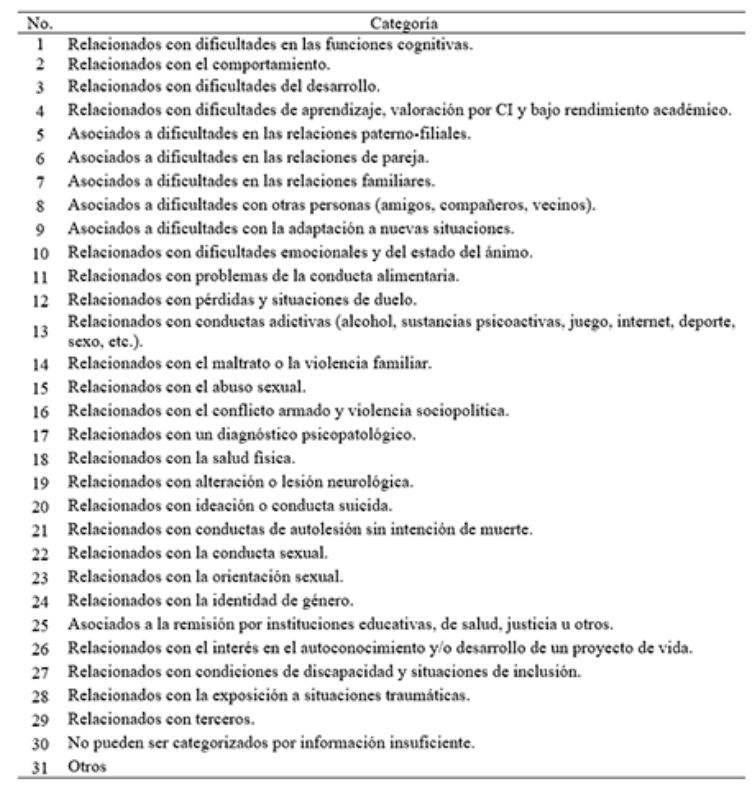

Teniendo en cuenta las diferencias entre las poblaciones, se solicitó adicionalmente, en el caso de los niños, el motivo de consulta reportado por el niño y también el manifestado por el adulto que lo acompañaba a la consulta. En las parejas, se realizaron preguntas orientadas a la vida en convivencia y su historia, tales como: asistencia previa a tratamiento psicológico de pareja, expectativas de la ayuda psicológica frente a la pareja, entre otras.

Diseño de artes y formato de las versiones finales del instrumento

Se diseñó un formato que permite, de forma sencilla y adecuada, el manejo, la implementación y el diligenciamiento del instrumento por parte del personal de los Centros. Esto implicó un trabajo de artes en diseño, estructuración y diagramación que permitió la transición del formato Excel en el que inicialmente se construyó el instrumento, a un formato en PDF que facilitó la logística de impresión de los formularios y la claridad visoespacial para su diligenciamiento.
Procedimiento

\section{Diseño de manual y material de apoyo}

Para favorecer la adecuada comprensión, apropiación e implementación del instrumento para su aplicación por parte de los Centros de atención psicológica, se diseñó un manual de aplicación que presenta el contexto, los objetivos y la relevancia del instrumento y la investigación que lo enmarca. También presenta las cuatro versiones del instrumento (adultos, parejas, adolescentes y niños); los cuatro ejes de caracterización (datos sociodemográficos, recursos psicosociales, riesgos psicosociales y motivos de consulta); las características poblacionales a las que responde cada versión del instrumento; el tipo de preguntas que se utilizan; las consideraciones y recomendaciones para el diligenciamiento de los formatos y aclaraciones sobre distintos términos técnicos utilizados en algunas preguntas y opciones de respuesta. El manual de aplicación contiene los formatos de consentimiento informado y un material de apoyo dirigido a las personas que responden el instrumento, para facilitar su comprensión, a medida que el entrevistador lee las preguntas.

\section{Capacitación en los Centros}

La transferencia del instrumento a los distintos Centros de Atención Psicológica Universitarios se llevó a cabo por medio de un proceso de capacitación en todas las regiones, el cual permitió dar a conocer el material y capacitar al personal para su adecuada implementación. En algunos Centros, esta capacitación se realizó a un grupo coordinador, cuyos integrantes fueron los encargados de transmitir la información a los demás estudiantes y profesionales en su institución.

El encuentro de capacitación permitió establecer acuerdos sobre la aplicación y la logística de envío de la información. En su desarrollo, se presentaron el marco y los objetivos de la investigación, el cronograma con las distintas fases del proyecto, el instrumento y 
sus distintas versiones, los aspectos legales y éticos que debían tenerse en cuenta en cada momento del proceso y la dinámica de envío y recepción de los formatos diligenciados. Lo anterior se complementó con ejercicios prácticos de aplicación del instrumento. Se entregaron los cuestionarios impresos, el manual y el material de apoyo de acuerdo con el promedio de nuevos consultantes recibidos mensualmente y reportado por los mismos, a todos los Centros participantes.

\section{Pilotaje en la IPS Consultores en Psicología}

Con el fin de dar inicio al pilotaje y aplicación del instrumento, se llevaron a cabo tres encuentros de capacitación a los estudiantes de pregrado y de Maestría en Psicología Clínica de la Facultad de Psicología de la Pontificia Universidad Javeriana, que realizaban su práctica en Consultores en Psicología y tendrían una participación en la aplicación del instrumento a la población consultante. Estas capacitaciones sirvieron para diseñar el manual de instrucciones, aclarar términos y reajustar preguntas y opciones de respuesta para que, en la aplicación, el personal encargado lo implementara adecuadamente.

\section{Aplicación del instrumento}

El terapeuta -estudiante bajo supervisión o profesional- consultaba a cada sujeto su disposición a participar en el estudio y le presentaba el consentimiento informado. Una vez realizado este procedimiento, se iniciaba la aplicación del cuestionario. Cada cuestionario fue diligenciado en papel, por el terapeuta. Un miembro del equipo designado en cada ISUAP como monitor, verificó el correcto diligenciamiento del formulario y gestionó el envío de los formularios al equipo central en Bogotá.

\section{Diseño de base de datos}

Personal capacitado hizo la digitación de los datos en una base de Excel@ ${ }^{\circledR}$, construida según los principios de "datos ordenados" o "tidy data” (Tierney \& Cook, 2020; Wickham, 2014), para facilitar el ingreso y tratamiento de la información en cualquier programa de análisis de datos. Para la digitación de los motivos de consulta, se dispuso de cuatro casillas: en la primera, se escribió lo que enunciaba el consultante y en la segunda, la categoría a la cual pertenecía; no obstante, si en la descripción se identificaba que el motivo podría clasificarse en más de una categoría, se registraba en la tercera y cuarta casillas, respectivamente, teniendo en cuenta la importancia dada por el paciente como motivo secundario. Dado que la categorización fue realizada por parejas de expertos en el área clínica, se implementó como método de convergencia la comparación de respuestas de los miembros de la pareja, es decir, el cálculo del porcentaje de consistencia o inconsistencia para posteriormente llegar a un acuerdo frente al motivo de consulta evaluado.

\section{Resultados}

El análisis descriptivo se llevó a cabo en SPSS® versión 25 , caracterizando con detalle la muestra en términos de los ejes del instrumento. El cuestionario se administró a 1525 personas en 28 Centros de Atención Psicológica Universitarios del país. El $56.9 \%$ de los cuestionarios fueron de adultos, $15.6 \%$ de adolescentes y 27.4 $\%$ de niños (Tabla 2). Se descartaron 39 formularios por errores en el diligenciamiento. Los errores identificados se encontraron en fechas de nacimiento del consultante que no correspondían con el tipo de cuestionario, en casillas de motivo de consulta vacías y más del 10 $\%$ de información incompleta o no suministrada. Por otra parte, de los 1525 cuestionarios, en 46 de ellos (30 en adultos, 9 en adolescentes y 7 en niños) una o dos preguntas quedaron sin respuesta. 
Tabla 2

Distribución de la población por grupos de edad en años

\begin{tabular}{|c|c|c|c|c|c|c|}
\hline \multicolumn{7}{|c|}{ Distribución de la población por grupos de edad en años } \\
\hline \multirow{2}{*}{$\begin{array}{l}\text { Grupo de edad } \\
\text { (en años) }\end{array}$} & \multicolumn{2}{|c|}{ Hombre } & \multicolumn{2}{|c|}{ Mujer } & \multicolumn{2}{|c|}{ Total } \\
\hline & Frecuencia & $\%$ & Frecuencia & $\%$ & Frecuencia & $\%$ \\
\hline 3 a 11 & 276 & 18 & 142 & 9.3 & 418 & 27.4 \\
\hline 12 a 17 & 110 & 7.2 & 128 & 8.3 & 238 & 15.6 \\
\hline 18 a 30 & 143 & 9.3 & 359 & 23.5 & 502 & 32.9 \\
\hline 31 a 60 & 114 & 7.4 & 224 & 14.6 & 338 & 22.1 \\
\hline 61 a 80 & 13 & 0.8 & 16 & 1 & 29 & 1.9 \\
\hline Total $(\mathrm{N}=1525)$ & 656 & 42.7 & 869 & 56.7 & 1525 & 100 \\
\hline
\end{tabular}

Fuente: Tomada de Colón-Llamas et al. (2019).

El interés del cuestionario desarrollado no fue la obtención de puntajes, la inferencia a partir de ellos ni el establecimiento de baremos, de modo que el proceso se enfocó en que el mecanismo de construcción de los ítems permitiera aumentar la validez de constructo y de contenido, a través de un trabajo sistemático y revisado de forma iterativa, para asegurar que los temas de estructura y formato correspondieran con lo que se deseaba indagar y de esta forma disminuir el riesgo de subrepresentar el constructo y sus dimensiones. Por lo anterior, se contó con la participación de equipos de profesionales con experiencia clínica y la revisión de la literatura previa como forma de evidenciar la validez de constructo. Asimismo, la posterior participación de jurados expertos fue crucial para asegurar la validez de contenido, relacionada con los temas, formato y escritura (American Educational Research Association et al., 2014).

\section{Conclusiones}

En la atención psicológica, la necesidad de un adecuado sistema de categorización y recopilación de motivos de consulta y factores psicosociales asociados es apremiante. Como centro de dicho sistema se propuso un instrumento construido específicamente para este propósito de categorización y recopilación, el cual fue utilizado para recoger información de más de 1000 consultantes en el año 2018, con el fin de validar su utilidad y producir información estadística sobre los motivos de consulta más frecuentes en el contexto colombiano y los factores psicosociales asociados a dicha consulta (Colón-Llamas et al., 2019).

El instrumento presentado se diseñó con base en un proceso de consulta en el cual participaron profesionales en psicología de diversas orientaciones terapéuticas, que trabajan en diferentes regiones del país. Estas consultas estuvieron informadas y retroalimentadas por expertos en metodologías de investigación, y produjeron un instrumento con una validez de contenido basada en la revisión bibliográfica y el juicio de expertos, que tal como ha sido señalado previamente, no solo se constituye a través de la selección adecuada de los ítems, sino también de elementos que enriquecen la aplicación del instrumento, como las instrucciones y el material de apoyo para su aplicación. Con ello, el cuestionario resulta exitoso para recoger información de una muestra grande de consultantes de Centros de Atención Psicológica Universitarios.

Por lo que se pudo constatar gracias a la revisión de literatura, un esfuerzo de tal sistematicidad y tan amplia participación es novedoso en Colombia y en la región latinoamericana. El proceso descrito se ofrece como insumo a equipos de otros países con el objetivo de orientar sus propios instrumentos adaptados a las características específicas de sus contextos, y estos puedan alimentar sistemas de recopilación de información. Asimismo, se pretende llamar la atención a la comunidad científica latinoamericana en torno a la importancia de emprender más estudios relacionados con la caracterización psicosocial y de los motivos de consulta, con el fin de estimar con ellos algunos índices de las propiedades psicométricas de los cuestionarios, desde la teoría de la generalizabilidad o de respuesta al ítem.

\section{Agradecimientos}

Las universidades aliadas y colaboradoras con el estudio fueron: Universidad de la Sabana, Universidad Santo Tomás, Universidad Católica de Colombia, Universidad del Bosque, Universidad Piloto de Colombia, 
Fundación los Libertadores, Universidad San Buenaventura Medellín, Universidad de Envigado, Universidad Pontificia Bolivariana Medellín, Universidad Católica de Oriente, Católica del Norte Fundación Universitaria, Universidad CES, Universidad de la Costa, Universidad del Norte, Universidad Metropolitana, Corporación Universitaria del Caribe, Universidad Surcolombiana, Universidad de Ibagué, Universidad Antonio Nariño Ibagué, Fundación de Estudios Superiores Monseñor Abraham Escudero Montoya (Espinal), Universidad Católica de Pereira, Universidad de Manizales, Universidad San Buenaventura (Armenia), Universidad Pontificia Bolivariana (Palmira), Universidad San Buenaventura (Cali), Universidad Pontificia Bolivariana (Bucaramanga).

\section{Referencias}

Ainsworth, P. (2000). Understanding depression. University Press of Mississippi. http://www.netLibrary.com/url api.asp? action $=$ summary $\& v=1 \&$ bookid $=$ 24993

American Educational Research Association, American Psychological Association, \& National Council on Measurement in Education. (2014). Standards for Educational and Psychological Testing. American Educational Research Association.

Asociación Médica Mundial. (21 de marzo de 2017). Declaración de Helsinki. Principios éticos para las investigaciones médicas en seres humanos. https://www.wma.net/es/policiespost/declaracion-de-helsinki-de-la-amm-pr incipios-eticos-para-las-investigaciones-me dicas-en-seres-humanos/

Beckham, E., \& Leber, W. (1997). Handbook of depression (2.. ed.). The Guilford Press.

Bezanilla, J. M., Miranda, M. A., Valdez, C., Viveros, M., Rivera, R., Botello, F., \& López, J. (febrero, 2017). Construcción de un modelo mexicano de atención psicosocial. Trabajo presentado en el XVIII Congreso
Virtual Internacional de Psiquiatría. http:// psiqu.com/1-7770

Cáceres, D., Salazar, I., Varela, M., \& Tovar, J. (2006). Consumo de drogas en jóvenes universitarios y su relación de riesgo y protección con los factores psicosociales. Universitas Psychologica, 5(3), 521-534. http://www.redalyc.org/articulo.o a? id $=64750308$

Campo-Arias, A., \& Caballero-Domínguez, C. C. (en prensa). Análisis factorial confirmatorio del cuestionario de APGAR familiar. Revista Colombiana de Psiquiatría. h ttps://doi.org/10.1016/j.rcp.2020.01.003

Clark, D. A., Beck, A. T., \& Alford, B. A. (1999). Scientific foundations of cognitive theory and therapy of depression. John Wiley \& Sons.

Colón-Llamas, V. T., Liemann Hernández, E., Marconi Martínez, L. I., Mateus Murillo, S. T., Nieto Sua, D. L., \& Sepúlveda, S. M. (2015). Caracterización de los centros universitarios de atención psicológica en Colombia. En Memorias I Congreso Colombiano de Psicología (pp. 245-246). Colegio Colombiano de Psicólogos. https://congresopsicologiacolo mbia.com/memorias/MEMORIAS_DEL_ CONGRESO 2015.pdf

Colón-Llamas, T., Escobar-Altare, A., Santacoloma-Giraldo, A., GranadosGarcía, A., Moreno-Luna, S., \& SilvaMartin, L. M. (2019). Caracterización psicosocial y de motivos de consulta de la población asistente a 28 Centros de Atención Psicológica Universitarios en Colombia. Universitas Psychologica, 18(4), 1-23. https://doi.org/10.11144/Javeriana.up sy18-4.cpmc

Corporación AVRE. (2002). Guía de orientaciones para atención en salud mental y trabajo psicosocial a población desplazada en Colombia. Autor.

de la Portilla Maya, S., Montoya-Londoño, D. M., Dussán-Lubert, C., \& López-Jaramillo, M. del R. (2016). Caracterización de los asistentes al Centro de Atención Psicológica de la Universidad de Manizales. Hacia La Promoción de La Salud, 21(2), 
127-141. https://doi.org/10.17151/hpsal.20 16.21.2.10

Escobar-Pérez, J., \& Cuervo-Martínez, A. (2008). Validez de contenido y juicio de expertos: una aproximación a su utilización. Avances en Medición, 6(1), 27|-36. https://www.rese archgate.net/publication/302438451_Vali dez_de_contenido_y_juicio_de_expertos_ Una_aproximacion_a_su_utilizacion

Espinosa Muñoz, M. C., Orozco Ramírez, L. A., \& Ybarra Sagarduy, J. L. (2015). Síntomas de ansiedad, depresión y factores psicosociales en hombres que solicitan atención de salud en el primer nivel. Salud Mental, 38(3), 201-208. https://doi.org/10. 17711/SM.0185-3325.2015.028

Galicia Alarcón, L., Balderrama Trápago, J., \& Navarro, R. (2017). Validez de contenido por juicio de expertos: propuesta de una herramienta virtual. Apertura, 9(2), 42-53. https://doi.org/10.32870/ap.v9n2.993

Gómez Vargas, M., \& Pérez Jaramillo, E. (2017). Particularidades de los motivos de consulta psicológica de los estudiantes que asisten a la Dirección de Bienestar Universitario, Universidad de Antioquia. Revista de Psicología Universidad de Antioquia, 9(1), 75-92. https://doi.org/10.17533/udea.rpsua .v9n1a06

González-Villalobos, J., \& Marrero, R. (2017). Determinantes sociodemográficos y personales del bienestar subjetivo y psicológico en población mexicana. Suma Psicológica, 24(1), 59-66. https://doi.org/10. 1016/j.sumpsi.2017.01.002

Hosey, M. M., Ali, M. K., Mantheiy, E. C., Albert, K., Wegener, S. T., \& Needham, D. M. (2019). Psychology consultation patterns in a medical intensive care unit: A brief report. Rehabilitation Psychology, 64(3), 360-365. https://doi.org/10.1037/rep00002 64

Hurtado-Parrado, C., Sierra Puentes, M. C., El Hazzouri, M., Morales, A., GutiérrezVillamarín, D., Velásquez, L., CorreaChica, A., Rincón, J. C., Henao, K., Castañeda, J. G., \& López-López, W. (abril, 2019). Emotion regulation and attitudes toward conflict in Colombia: Effects of reappraisal training on negative emotions and support for conciliatory and aggressive statements. Frontiers in Psychology. https://d oi.org/10.3389/fpsyg.2019.00908

Jellinek, M. S., Murphy, J. M., Robinson, J., Feins, A., Lamb, S., \& Fenton, T. (1988). Pediatric symptom checklist: Screening school-age children for psychosocial dysfunction. The Journal of Pediatrics, 112 (2), 201-209. https: //doi.org/10.1016/S0022-3476(88)80056-8

Londoño, N. H., Marín, C. A., Juárez, F, \& Palacio, J. (2010). Factores de riesgo psicosociales y ambientales asociados a trastornos mentales. Suma Psicológica, 17(1), 59-68. http://www.scielo.org.co/pdf/ sumps/v17n1/v17n1a5.pdf

López Jaramillo, M. del R., Montoya Londoño, D. M., \& Dussán Lubert, C. (2012). Caracterización de los asistentes al Centro de Atención Psicológica de la Universidad de Manizales, 2006-2010. Hacia La Promoción de La Salud, 17(2), 149-166. htt ps://doi.org/10.17151/hpsal.2016.21.2.10

López López, W., Sandoval Alvarado, G., Rodríguez, S., Ruiz, C., León, J. D., PinedaMarín, C., \& Mullet, E. (2018). Forgiving former perpetrators of violence and reintegrating them into Colombian civil society: Noncombatant citizens' positions. Peace and Conflict: Journal of Peace Psychology, 24(2), 201-215. https://doi.org/ 10.1037/pac0000295

Maroto-Vargas, A., Molina-Fallas, L., \& Prado-Calderón, J. (2017). Características sociodemográficas y motivos de consulta de las personas atendidas en el Centro de Atención Psicológica de la Universidad de Costa Rica (2004-2013). Revista Costarricense de Psicología, 36(1), 23-44. htt ps://doi.org/10.22544/rcps.v36i01.02

Martínez Ramos, M. E., \& Pacheco García, O. E. (2013). Utilidad de los Registros Individuales de Prestación de Servicios (RIPS) para la vigilancia en salud pública, Colombia, 2012 (Informe quincenal epidemiológico nacional). Instituto Nacional de Salud. 
Miguel-Álvaro, A., \& Fernández-Arias, I. (2019). ¿Coincide la demanda del paciente con el problema percibido por el terapeuta? Un análisis cualitativo de motivos de consulta. Revista Argentina de Clínica Psicológica, 28(4), 363-374. https://doi.org/ 10.24205/03276716.2019.1143

Miller, L. A., \& Lovler, R. L. (2020). Foundations of psychological testing: A practical approach. Sage.

Ministerio de Salud y Protección Social $\&$ Colciencias. (2015). Encuesta nacional de salud mental 2015. Autor. http://www.odc.gov.co/Portals/1/pu blicaciones/pdf/consumo/estudios/nacional es/CO031102015-salud_mental_tomoI.pdf Ministerio de Salud y Protección Social. (2017). Protocolo de atención integral en salud con enfoque psicosocial. Autor. https://www.mins alud.gov.co/sites/rid/Lists/BibliotecaDigital /RIDE/DE/PS/Protocolo-de-atencion-integ ral-en-salud-papsivi.pdf

Moreno Méndez, J. H., Rozo Sánchez, M. M., Cantor Nieto, M. I., \& Toro Tobar, R. A. (2012). Características psicosociales de la población asistente a la unidad de servicios psicológicos de la Universidad Católica de Colombia durante los años 2007 y 2008. Psicología. Avances de la Disciplina, 6(1), 21-33. http://www.scielo.org.co/scielo.php? script $=$ sci arttext\&pid $=$ S1900-23862012 000100003 \& $\operatorname{lng}=$ en\& $\&$ rm $=$ iso\&tlng $=\mathrm{es}$

Narváez Marín, N., \& Aguirre Loaiza, H. (2016). Análisis de las impresiones diagnósticas en consultantes de un Centro de Atención Psicológica Universitario. Informes Psicológicos, 16(2), 87-101. https:// doi.org/10.18566/infpsicv16n2a06

Obando-Posada, D., Romero-Porras, J., TrujilloCano, A., \& Prada-Mateus, M. (2017). Estudio epidemiológico de salud mental en población clínica de un centro de atención psicológica. Psychologia, 11(1), 85-96. http://www.scielo.org.co/pdf/psych/ v11n1/1900-2386-psych-11-01-00085.pdf

Pineda, D. A., Lopera, F., Palacio, J. D., Ramírez, D., \& Henao, G. C. (2003). Prevalence estimations of attention- deficit/hyperactivity disorder: Differential diagnoses and comorbidities in a Colombian sample. International Journal of Neuroscience, 113(1), 49-71. https://doi.org /10.1080/00207450390161921

Quesada, S. (2004). Estudio sobre los motivos de consulta psicológica en una población universitaria. Univesitas Psychologica, 3(1), 7-16. https://www.redalyc.org/pdf/647/647 30102.pdf

Smilkstein, G. (1978). The Family APGAR: A proposal for a family function test and its use by physicians. The Journal of Family Practice, 6(6), 1231-1239. https://psycnet.a pa.org/record/1979-26481-001

Sosa, J., Romero, M., Blum, B., Zarco, V., \& Medina-Mora, M. (2018). Programa de orientación y atención psicológica para jóvenes universitarios de la UNAM: características de la población que solicita sus servicios. Revista Electrónica de Psicología Iztacala, 21(2), 451-477. https://www.iztacala.unam.mx/ca rreras/psicologia/psiclin/vol21num2/Vol21 No2Art5.pdf

Tierney, N., \& Cook, D. (2020). Expanding tidy data principles to facilitate missing data exploration, visualization and assessment of imputations. Journal of Statistical Software, 20(2), 1-30. https://doi.org/10.18637/jss.v0 $00 . \mathrm{i} 00$

Wickham, H. (2014). Tidy Data. Journal of Statistical Software, 59(10). https://doi.org/1 0.18637/jss.v059.i10

World Medical Association. (2017). Declaración de Helsinki de la AMM - Principios éticos para las investigaciones médicas en seres humanos. Autor. http://www.redsamid.net/archivos/2 01606/2013-declaracion-helsinki-brasil.pdf ?1

\section{Notas}

* Artículo de investigación. Este estudio fue financiado por la Pontificia Universidad Javeriana, sede Bogotá, a través de Proyectos de Contribución de las facultades en el marco de la Planeación Universitaria 2021. 